\title{
Assessment of Carbon Substrate Catabolism Pattern and Functional Metabolic Pathway for Microbiota of Limestone Caves
}

\author{
Suprokash Koner ${ }^{1,2,+}$, Jung-Sheng Chen ${ }^{3,+} \mathbb{D}$, Bing-Mu Hsu ${ }^{2,4, * \mathbb{D}}$, Chao-Wen Tan ${ }^{5,+}$, Cheng-Wei Fan ${ }^{2}$, \\ Tsung-Hsien Chen ${ }^{6} \mathbb{D}$, Bashir Hussain ${ }^{1,2} \mathbb{D}$ and Viji Nagarajan ${ }^{2}$
}

Citation: Koner, S.; Chen, J.-S.; Hsu, B.-M.; Tan, C.-W.; Fan, C.-W.; Chen, T.-H.; Hussain, B.; Nagarajan, V. Assessment of Carbon Substrate Catabolism Pattern and Functional Metabolic Pathway for Microbiota of Limestone Caves. Microorganisms 2021, 9, 1789. https://doi.org/ 10.3390/microorganisms 9081789

Academic Editors: Tsing Bohu,

Xuliang Zhuang and

Ignacio Gonzalez-Alvarez

Received: 26 July 2021

Accepted: 19 August 2021

Published: 23 August 2021

Publisher's Note: MDPI stays neutral with regard to jurisdictional claims in published maps and institutional affiliations.

Copyright: (c) 2021 by the authors. Licensee MDPI, Basel, Switzerland. This article is an open access article distributed under the terms and conditions of the Creative Commons Attribution (CC BY) license (https:// creativecommons.org/licenses/by/ $4.0 /)$.
1 Department of Biomedical Sciences, National Chung Cheng University, Chiayi City 621, Taiwan; suprokashkoner@alum.ccu.edu.tw (S.K.); bashir.aku@gmail.com (B.H.)

2 Department of Earth and Environmental Sciences, National Chung Cheng University, Chiayi City 621, Taiwan; cwfan@eq.ccu.edu.tw (C.-W.F.); mathumitha08@gmail.com (V.N.)

3 Department of Medical Research, E-Da Hospital, Kaohsiung 824, Taiwan; nicky071214@gmail.com

4 Center for Innovative on Aging Society (CIRAS), National Chung Cheng University, Chiayi City 621, Taiwan

5 Division of Cardiology, Department of Internal Medicine, Ditmanson Medical Foundation Chiayi Christian Hospital, Chiayi City 600, Taiwan; kobebrnt2000@gmal.com

6 Department of Internal Medicine, Ditmanson Medical Foundation Chiayi Christian Hospital, Chiayi City 600, Taiwan; cych13794@gmail.com

* Correspondence: bmhsu@ccu.edu.tw; Tel.: +886-5272-0411 (ext. 66218)

+ Contributed equally to this work.

Abstract: Carbon utilization of bacterial communities is a key factor of the biomineralization process in limestone-rich curst areas. An efficient carbon catabolism of the microbial community is associated with the availability of carbon sources in such an ecological niche. As cave environments promote oligotrophic (carbon source stress) situations, the present study investigated the variations of different carbon substrate utilization patterns of soil and rock microbial communities between outside and inside cave environments in limestone-rich crust topography by Biolog EcoPlate ${ }^{\mathrm{TM}}$ assay and categorized their taxonomical structure and predicted functional metabolic pathways based on $16 \mathrm{~S}$ rRNA amplicon sequencing. Community level physiological profiling (CLPP) analysis by Biolog EcoPlate ${ }^{\mathrm{TM}}$ assay revealed that microbes from outside of the cave were metabolically active and had higher carbon source utilization rate than the microbial community inside the cave. $16 \mathrm{~S}$ rRNA amplicon sequence analysis demonstrated, among eight predominant bacterial phylum Planctomycetes, Proteobacteria, Cyanobacteria, and Nitrospirae were predominantly associated with outside-cave samples, whereas Acidobacteria, Actinobacteria, Chloroflexi, and Gemmatimonadetes were associated with inside-cave samples. Functional prediction showed bacterial communities both inside and outside of the cave were functionally involved in the metabolism of carbohydrates, amino acids, lipids, xenobiotic compounds, energy metabolism, and environmental information processing. However, the amino acid and carbohydrate metabolic pathways were predominantly linked to the outside-cave samples, while xenobiotic compounds, lipids, other amino acids, and energy metabolism were associated with inside-cave samples. Overall, a positive correlation was observed between Biolog EcoPlate ${ }^{\mathrm{TM}}$ assay carbon utilization and the abundance of functional metabolic pathways in this study.

Keywords: microbial community; Biolog EcoPlate ${ }^{\mathrm{TM}}$ assay; 16S RNA amplicon; hylogenetic investigation of communities by reconstruction of unobserved states (PICRUSt); functional metabolic pathway prediction; limestone

\section{Introduction}

Microbes-associated biomineralization is a widespread phenomenon in regions rich in limestone, and ultimately leads to the precipitation of calcium carbonate [1]. In this 
process, the available nucleation sites are one of the prime governing factors along with the concentration of calcium ions, dissolved inorganic matter, and the $\mathrm{pH}$ of that particular atmosphere [2]. As such, the growth and metabolic activity of microbes can ubiquitously speed up the biomineralization process by providing more nucleation sites for $\mathrm{CaCO}_{3}$ precipitation in such an environment [3,4]. The efficiency of exogenous carbon source utilization from soil organic carbon (SOC) could be an embracive signature of microbial community metabolic characteristics and their growth [5]. Thus, previous studies reported that the growth of bacteria cells and their metabolism are strongly influenced by diverse carbon source catabolism activity, because it may deliver the building block metabolites and energy for them [6,7]. In this aspect, the pool of SOC might be different according to the variations of natural earth crust structures, which are promotionally allied with the amount of soil microbial biomass [8,9]. However, the assimilation or uptake of carbon from the environment is considered the key factor for microbial-mediated calcification in limestone [10].

In terrestrial limestone-rich habitats, the majority of heterotopic calcite-precipitated bacteria play a role in the carbon cycle via the utilization of soil organic matter (SOM) from organic matter [11]. Based on its molecular mass, SOM can be traditionally categorized as either low-molecular-weight (LMW) or high-molecular-weight (HMW) [12]. Furthermore, carbohydrates, organic/amino acids, proteins, siderophores, lipids, phenolics, hormones, and vitamins, among others, can be categorized into different classes of LMW compounds, and have relatively less persistence in terrestrial ecosystems due to their higher uptake and metabolisms by soil microbiota [13]. These are formed during the partial decomposition and conversion of plant inputs, such as root exudates and upper and lower-ground litter by soil organisms [14]. Caves are considered extreme environments due to the absence of sunlight. Here, no photosynthesis occurs, resulting in oligotrophic conditions [15]. Nonphotosynthetic primary production is always carried out by several chemoautotrophic microbes and supporting other bacteria groups to be sustained [16]. The previous report regarding such an environment emphasized that some heterotopic bacteria, which might be in the presence of $\mathrm{Ca}$ ions and $\mathrm{CO}_{2}$, can construct calcium carbonate via decomposition of urea by urease enzyme $[17,18]$. In such nutrient-limited ecosystems, a small amount of allochthonous carbon can accumulate with the help of surface runoff and vadose-zone leaching from photic surface environments, including macrofaunal activity, which act as pioneer organic matter sources, leading to the microbial biomineralization of calcium carbonate $[19,20]$.

As substrates of Biolog EcoPlate ${ }^{\mathrm{TM}}$ also belong to LMW carbon compounds, it would be a promising technique and powerful analytical tool to determine the carbon catabolism ability outlook of heterotopic bacteria during their cell growth process in plate conditions [21]. This approach characterizes the metabolic diversity of environmental samples using community-level physiological profiling (CLPP) $[22,23]$. The Biolog EcoPlate ${ }^{\mathrm{TM}}$ assay plate consists of 31 most-useable carbon sources in triplicate order with tetrazolium redox violet dye; during each substrate utilization through the inoculated microbiota community, the dye converts into a purple color and denotes as subsequent carbon soles are utilized [24]. Next-generation sequencing (NGS) is a revolutionary breakthrough, and uses present decays as a robust culture-independent technique to explore the biodiversity of complex environmental niches based on 16S rRNA targeting specific regions of DNA sequences [25]. This 16S rRNA gene amplicon sequence is used to obtain prediction-based phylogenetic structures of the uncultivated microbial community, metabolic pathway, functional genes, and species diversity studies [26]. The phylogenetic investigation of communities by the reconstruction of unobserved states (PICRUSt) analysis is a computational approach to predict the functional potentiality of microbial groups based on 16S rRNA hypervariable amplicon sequencing data. A reference genomic database was generally linked with PICRUSt analysis to predict the functional role of unculturable prokaryotes in a precise ecosystem $[27,28]$. Together with Biolog EcoPlate ${ }^{\mathrm{TM}}$ substrate utilization, $16 \mathrm{~S}$ 
rRNA amplicon-based functional prediction data would be a compact approach to explore the overall view on the microbial community's metabolic fingerprint.

Organic matter has been reported to influence the microbial community associated with biomineralization by several bacterial species [1]. Still, there is a lacuna on how it can affect upon a microbiome's communal metabolic fingerprint in such an environment. Currently, no studies have characterized the microbiota inside and outside of caves in a limestone-rich region using a community-level physiological profiling (CLPP) approach in combination with $16 \mathrm{~S}$ rRNA amplicon-based functional prediction analysis. In the present study, the carbon catabolism patterns of microbial communities inside and outside a limestone cave, as well as their functional diversity from a holistic point of view, were investigated. To this end, the Biolog EcoPlate ${ }^{\mathrm{TM}}$ assay was used to determine the carbon assimilation ability of limestone microbiota. Additionally, NGS was applied to the taxonomic structure of the microbial community, and PICRUSt2 analysis was performed using the KEGG (Kyoto Encyclopedia of Genes and Genomes) reference database to predict the metabolic pathways of the microbial communities studied.

\section{Materials and Methods}

\subsection{Sample Collection Information}

The sampling site was located in the Tianliao district of Southern Taiwan. Rock and soil samples were obtained from two different zones of the limestone-rich region, according our sampling criteria: outside a limestone cave $\left(22^{\circ} 50^{\prime} 40^{\prime \prime} \mathrm{N} ; 120^{\circ} 23^{\prime} 10^{\prime \prime} \mathrm{E}\right)$ and inside a limestone cave $\left(22^{\circ} 50^{\prime} 40^{\prime \prime} \mathrm{N} ; 120^{\circ} 23^{\prime} 10^{\prime \prime} \mathrm{E}\right)$, shown in Figure 1 . In addition, specific meteorological parameters, such as solar radiation intensity, ambient temperature, humidity, and soil temperature, from the region of sample collection were recorded. Details of the sampling information are provided in Supplementary Table S1. The rock samples were collected from the surface area of the mother rock and stored in a pre-sterilized zipper bag to avoid contamination. The soil samples were collected from beneath the topsoil $(\mathrm{O}$ horizon) zone corresponding to the rock samples collected from the surrounding area and stored as above. Ultimately, all samples were transported directly to the laboratory using a temperature-controlled box.

\subsection{Carbon Substrate Utilization Pattern}

Community-level carbon substrate utilization patterns were determined for all soil and rock samples using a commercial phenotyping microarray tool (Biolog EcoPlate ${ }^{\mathrm{TM}}$ Inc., Hayward, CA, USA). For sample pre treatment, an improved version of the experimental protocol was used, according to the manufacturer's instructions. Briefly, $2 \mathrm{~g}$ of soil and rock samples were taken separately into 30-mL sterilized conical tubes, followed by $10 \mathrm{~min}$ of vortexing with $19 \mathrm{~mL}$ of $1 X$ sterilized phosphate buffer solution (PBS). The sample tubes were mixed homogeneously with PBS by shaking for up to $1 \mathrm{~h}$ to release bacterial cells, followed by $10 \mathrm{~min}$ of holding under static conditions to allow the soil particles to settle down. Then, $2 \mathrm{~mL}$ of supernatant was taken carefully and a serial dilution of up to 10-3-fold was performed to reduce the microbial load to obtain an efficient result and consider similar cell concentration in each sample. Finally, $100 \mu \mathrm{L}$ of suspension from the 10-3-fold dilution tube was inoculated into each well of a Biolog microplate and incubated under dark conditions at $25^{\circ} \mathrm{C}$ for $168 \mathrm{~h}$. A suspension of pure cultured E. coli with the same dilution as the sample was used as a positive control, while double-distilled water was used as a negative control, and inoculated into the corresponding microplate wells.

To determine the substrate utilization pattern of the microbial communities sampled, the optical density (OD) of each incubated microplate was observed every $24 \mathrm{~h}$ at a wavelength of $590 \mathrm{~nm}$ using a microplate reader (Multiskan ${ }^{\mathrm{TM}}$ FC Microplate Photometer; Thermo Scientific, Loughborough, UK) [22]. Mathematical equations were used to calculate the average well color development (AWCD) for each microplate, according to a previous study [29]. The OD readings against each well were corrected using the OD reading of water as a control. After correction, the negative readings were adjusted to zero. Lastly, 
based on substrate utilization patterns by microbes, the substrate utilization Shannon diversity substrate richness $(\mathrm{H})$ and evenness $(\mathrm{D})$ were calculated, according to previous studies $[21,24,30]$.
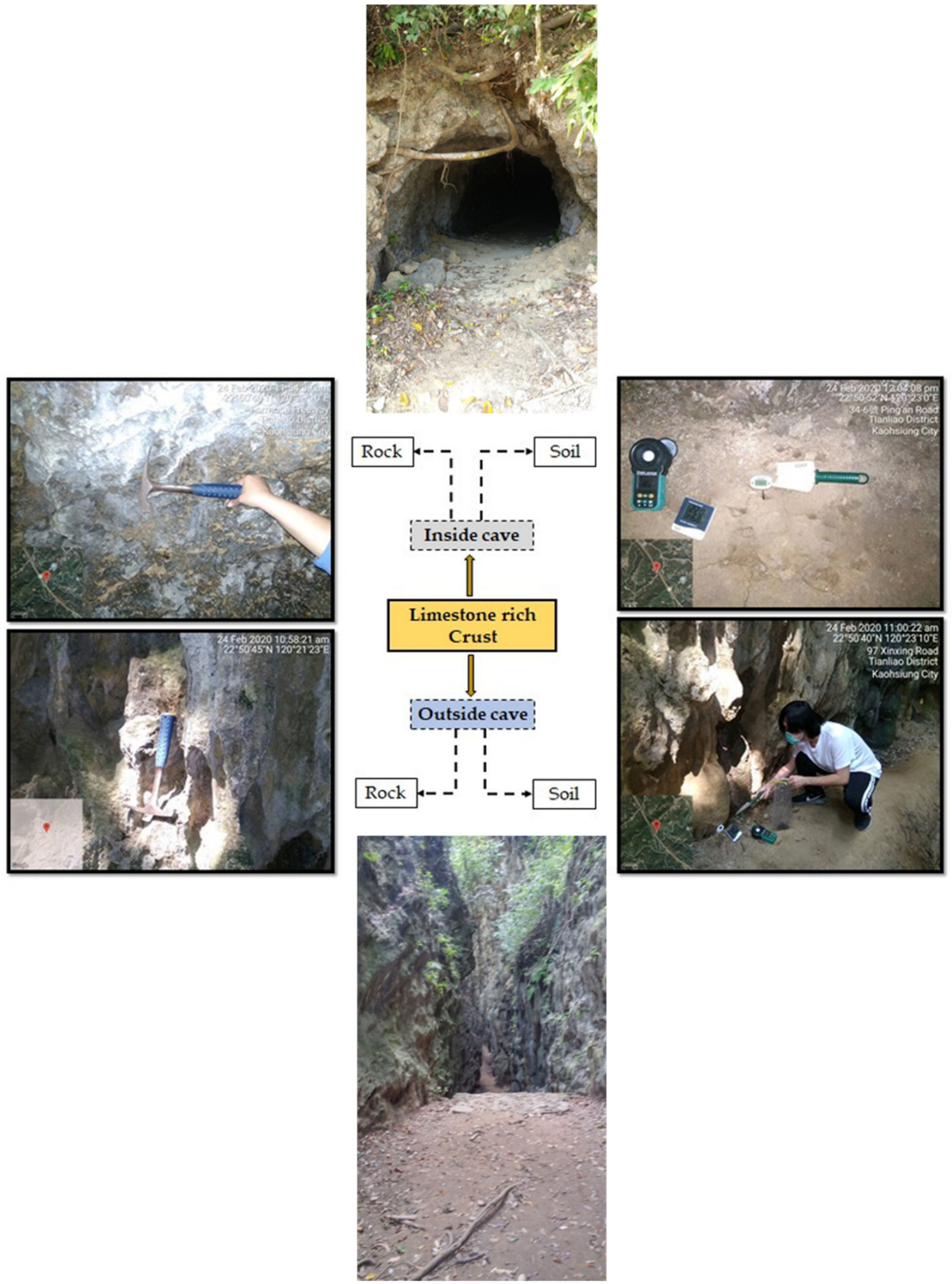

Figure 1. Sampling area and site description. 


\subsection{Microbial Genomic DNA Extraction}

In order to obtain homogenized microbial genomic DNA, each sample was evenly mixed, and large particles were segregated using a sieve to ensure complete homogenization and DNA extraction. Then, $0.5 \mathrm{~g}$ of fine powder from the rock and soil samples were inoculated into respective lysis tubes for the extraction of gDNA as per the protocol of NucleoSpin ${ }^{\circledR}$ Soil, a commercial kit for gDNA extraction (MACHEREY-NAGEL GmbH \& Co., Düren, Germany), with some modifications: instead of single step elution, two elution times were performed using $50 \mu \mathrm{L}$ of SE buffer, followed by $1 \mathrm{~min}$ incubation and $30 \mathrm{~s}$ of vortexing to obtain the maximum volume of DNA. Finally, the quantification and quality of gDNA was assessed at 260/280 wavelength using a Nanodrop 1000 (Thermo Fisher Scientific, Waltham, MA, USA). The extracted gDNA was stored at $-20{ }^{\circ} \mathrm{C}$ until further NGS analysis.

\subsection{S rRNA Amplicon Library Preparation and Functional Prediction}

The amplification of the 16S rRNA gene targeting V3-V4 regions gene sequence was carried out by using the KAPA HiFi HotStart Ready Mix PCR kit, and primer information is shown in Supplementary Table S4. Amplicon PCR amplification was performed using Illumina's MiSeq system (Illumina, San Diego, CA, USA) following a comprehensive protocol for paired-end sequencing, as described in a previous study [31]. After obtaining the raw sequencing files from the NGS platform, the data were further analyzed using the Qiime2 software package to characterize the microbial diversity at the phylum and genus levels [32]. In short, the sequence data were trimmed to remove chimera and clustered into Amplicon Sequence Variant (ASV) using a 97\% threshold limit of similarity with respect of Greengenes database. Lastly, the Qiime2 view was used to determine the bacterial composition of each sample. Additionally, PICRUSt2 software based on the Kyoto Encyclopedia of Genes and Genomes (KEGG) was used to predict the functional pathways associated with the microbial taxa within each community, as described previously (http:/ / picrust.github.io/picrust/, accessed on 1 May 2020) [33].

\subsection{Data Visualization and Statistical Analysis}

The Student T-test was used to show a statistically significant difference between outside and inside-cave microbiota substrate utilization AWCD value, Shannon index $\left(\mathrm{H}^{\prime}\right)$, Simpson index (D), and Shannon evenness score in soil and rock samples. Additionally, Pearson's correlation analysis was performed using SPSS (https://www.ibm. $\mathrm{com} /$ products/spss-statistics, accessed on 5 May 2020) to determine the relationship between carbon source utilization and abundance of predicted functional pathway reads of the microbial communities. To determine the association between bacterial taxonomies and functional predictions, heatmaps with dendrograms were computed using the webbased tool clustvist (https:/ / biit.cs.ut.ee/clustvis/, accessed on 10 May 2020) and a morpheus heatmap (https://software.broadinstitute.org/morpheus/, accessed on 13 May 2020). Principal component analysis (PCA) was conducted using canoco5.1 software (http:/ / www.canoco5.com/, accessed on 14 May 2020). The variations in the functional metabolic pathways between each sample after PICRUSt2 analysis were visualized using excel 2007 accessed on 14 May 2020.

\section{Results}

\subsection{Variation in Average Well Color Development ( $A W C D$ ) during Incubation Period}

The average well color development (AWCD) curve was plotted during the incubation period after the inoculation of the samples into the Biolog EcoPlate ${ }^{\mathrm{TM}}$, as shown in Figure 2. The microbial communities of the samples collected from outside the cave (LR-01 and LS-01) exhibited the highest AWCD kinetics, suggesting that the microbiota of these samples were highly active in their use of different types of carbon sources during cell growth. Besides a higher substrate utilization, Shannon diversity index $(\mathrm{H})$ were found in this area's samples at the end of the incubation period (168 h), shown in Table 1 . The microbial community 
in this area also indicated that they have a shorter lag phase period of cell growth. In addition, substrate utilization Shannon evenness scores in these samples were 0.86 and 0.97 (Table 1). Conversely, rock and soil samples from inside the cave showed lower AWCD rates than those collected outside of the cave $(p<0.05)$. This suggests that the microbial community in this sampling zone was lacked the activity to utilize different types of external carbon sources during cellular growth, resulting in a longer lag phase, as observed in both samples. In particular, the rock microbiota sample required around $96 \mathrm{~h}$ to consume a significant amount of Biolog EcoPlate ${ }^{\mathrm{TM}}$ substrates compared with the soil sample, which was markedly faster (24-48 h). Furthermore, substrate utilization Shannon diversity index and evenness scores of both the rock and soil samples in this area were low $(H=2.17$, 2.65; Shannon evenness $=0.62,0.76 ; p<0.05$ ). This analogous trend of carbon substrate utilization between different sampling zones suggests that microclimate conditions have an effect on the carbon source assimilation pattern of microbial communities. Additionally, the positive control showed a certain AWCD value during the incubation period, while no AWCD value was found for the negative control.

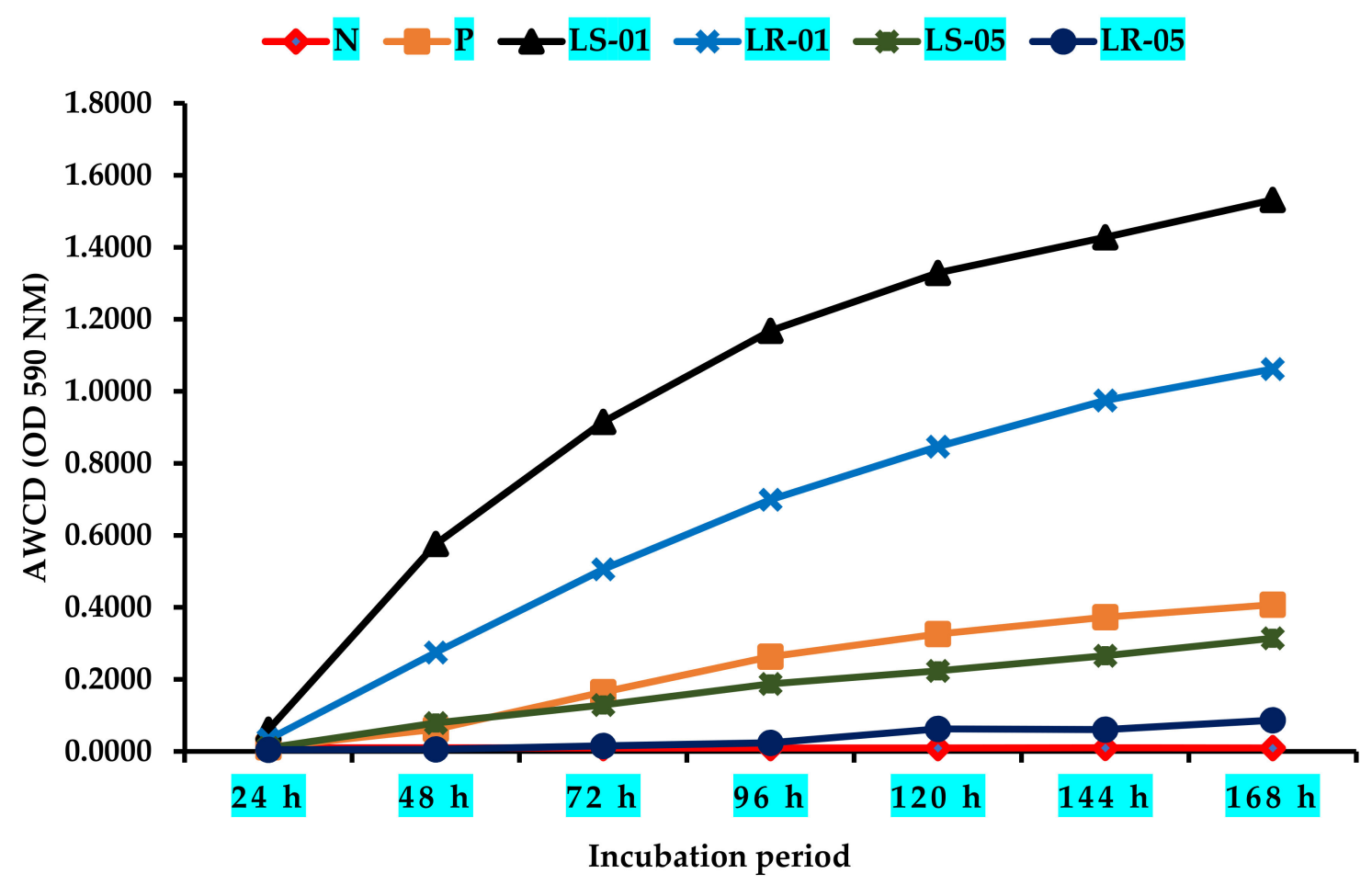

Figure 2. Changes in AWCD cure of all rock and soil samples according to different sample zones (LS1 and LR1, outside cave; LS5 and LR5, inside cave). $\mathrm{N}=$ negative control, $\mathrm{P}=$ positive control.

Table 1. Substrate utilization diversity and evenness index among rock and soil samples according to the different sampling zones.

\begin{tabular}{cccccc}
\hline Parameters & LR-01 & LS-01 & LR-05 & LS-05 & $p$ Value \\
\hline AWCD & 1.06 & 1.53 & 0.09 & 0.31 & $<0.05$ \\
Shannon & 3.00 & 3.38 & 2.18 & 2.65 & $<0.05$ \\
$\begin{array}{l}\text { Index H' } \\
\text { Simpson }\end{array}$ & 0.98 & 0.98 & 1.00 & 0.98 & $>0.05$ \\
$\begin{array}{l}\text { Index D } \\
\text { Shannon }\end{array}$ & 0.87 & 0.98 & 0.63 & 0.77 & $<0.05$ \\
evenness & & & & &
\end{tabular}

LR-01, LS-01: outside the cave; LR-05, LS-05: inside the cave. 


\subsection{Major Carbon Source Assimilation Pattern at End Point (168 h)}

The 31 types of carbon substrates found in Biolog EcoPlate ${ }^{\mathrm{TM}}$ were grouped into six major categories according to their biochemical properties: carbohydrates, amino acids, carboxylic acids, polymers, amines, and phenols. The utilization capability of these six categories, which was highest in the samples from outside the cave compared with the samples taken from inside the cave, where the utilization rate was quite low, is shown in Figure 3. The microbial communities in the rock and soil samples collected from outside the cave were found to utilize carbohydrates, amino acids, carboxylic acid, and polymers more commonly than amines and phenols. However, the higher degree of carbohydrate and carboxylic acid utilization was more closely associated with the soil microbiota than the rock microbiota. By contrast, although the microbial communities of the rock and soil samples collected from inside the cave initially consumed less of the major groups of carbon substrates, they showed a considerable utilization at the end of the incubation period $(168 \mathrm{~h})$, indicating that these microbes are also able to catabolize carbohydrates, amino acids, carboxylic acids, and polymers with a significantly slower utilization rate during cellular growth. Furthermore, the microbial communities of the soil samples showed a faster rate of amino acid, carbohydrate, and carboxylic acid assimilation than the rock samples inside the cave, followed by amines and phenols. Notably, polymers were used up more by the microbiota of the rock samples than the microbiota of the soil samples inside the cave.

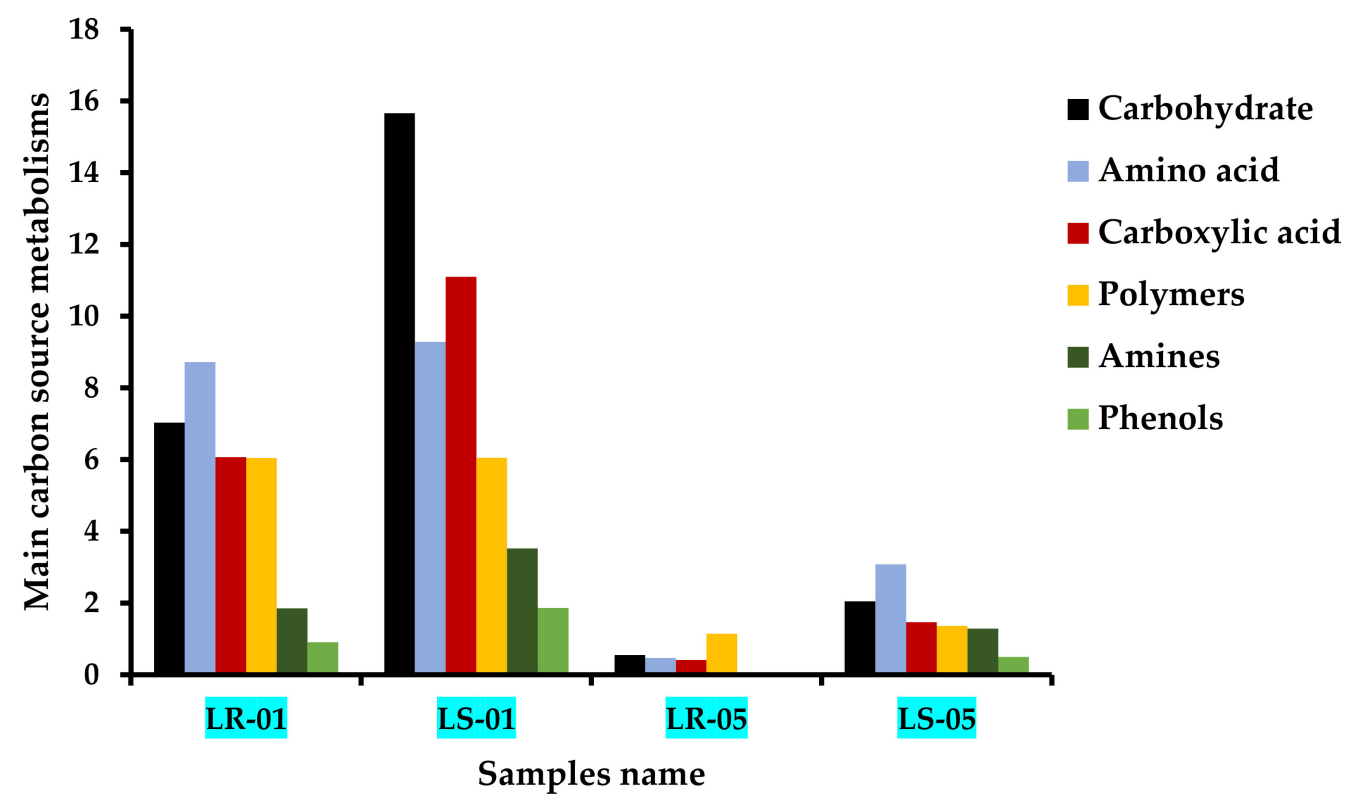

Figure 3. Major carbon source metabolic patterns at the incubation end point ( $168 \mathrm{~h}$ ) of all rock and soil samples according to the different sampling zones (LS1 and LR1, outside cave; LS5 and LR5, inside cave).

\subsection{Analysis of Bacterial Community Compositions and Distribution Patterns Using $16 S$ rRNA Amplicon-Based Techniques}

The bacterial compositions of the microbiota of the soil and rock samples both outside and inside the cave are shown in Figure 4 at the phylum level. A total of 27 classified taxa at phylum level were detected using 16S rRNA amplicon data across all samples, with similar beta diversity patterns (Supplementary Figure S1 and Supplementary Table S5). Among these, Proteobacteria, Acidobacteria, Actinobacteria, and Planctomycetes were the most predominant, followed by Nitrospirae, Chloroflexi, and Gemmatimonadetes in all of the samples, except Cyanobacteria, which was only present in the samples collected outside the cave. However, the relative abundance of Cyanobacteria was higher in the soil samples $(9.33 \%)$ than the rock samples $(2.59 \%)$. The relative abundance of Proteobacteria, 
Planctomycetes, Actinobacteria, and Acidobacteria was $12.75 \%$ and $13.60 \%, 26.61 \%$, and $32.07 \%, 18.23 \%$ and $6.94 \%$, and $8.08 \%$ and $13.05 \%$ in the samples collected from rock and soil outside the cave, respectively. By comparison, the relative abundance of Actinobacteria, Acidobacteria, and Proteobacteria were $26.98 \%$ and $21.37 \%, 17.68 \%$ and $17.81 \%$, and $21.97 \%$ and $21.67 \%$ in the rock and soil samples collected inside the cave, respectively. Pearson's correlation test was used to determine the effect of key environmental factors (e.g., sunlight, humidity, and temperature) on the distribution pattern of the bacteria identified. Planctomycetes, Proteobacteria, and Cyanobacteria were found to be significantly positively correlated with sunlight, while humidity was found to be negatively correlated (Supplementary Table S2). Actinobacteria showed an inverse relationship with sunlight and humidity compared with the abovementioned phyla. Dendrogram plot was constructed using a heatmap to visualize the distribution pattern of the bacteria at the genus level (Supplementary Figure S2). As a result, two separates of clusters were observed, each denoting one of the two different zones of our sampling criteria: cluster 1 was found to be closely related to the bacterial genus diversity of the rock and soil samples collected from the inside cave, and cluster 2 represented the genus level distribution of the microbiota of the rock and soil samples collected from outside the cave. Thus, the different types of sampling zones showed similar types of bacterial genus diversity distribution patterns.

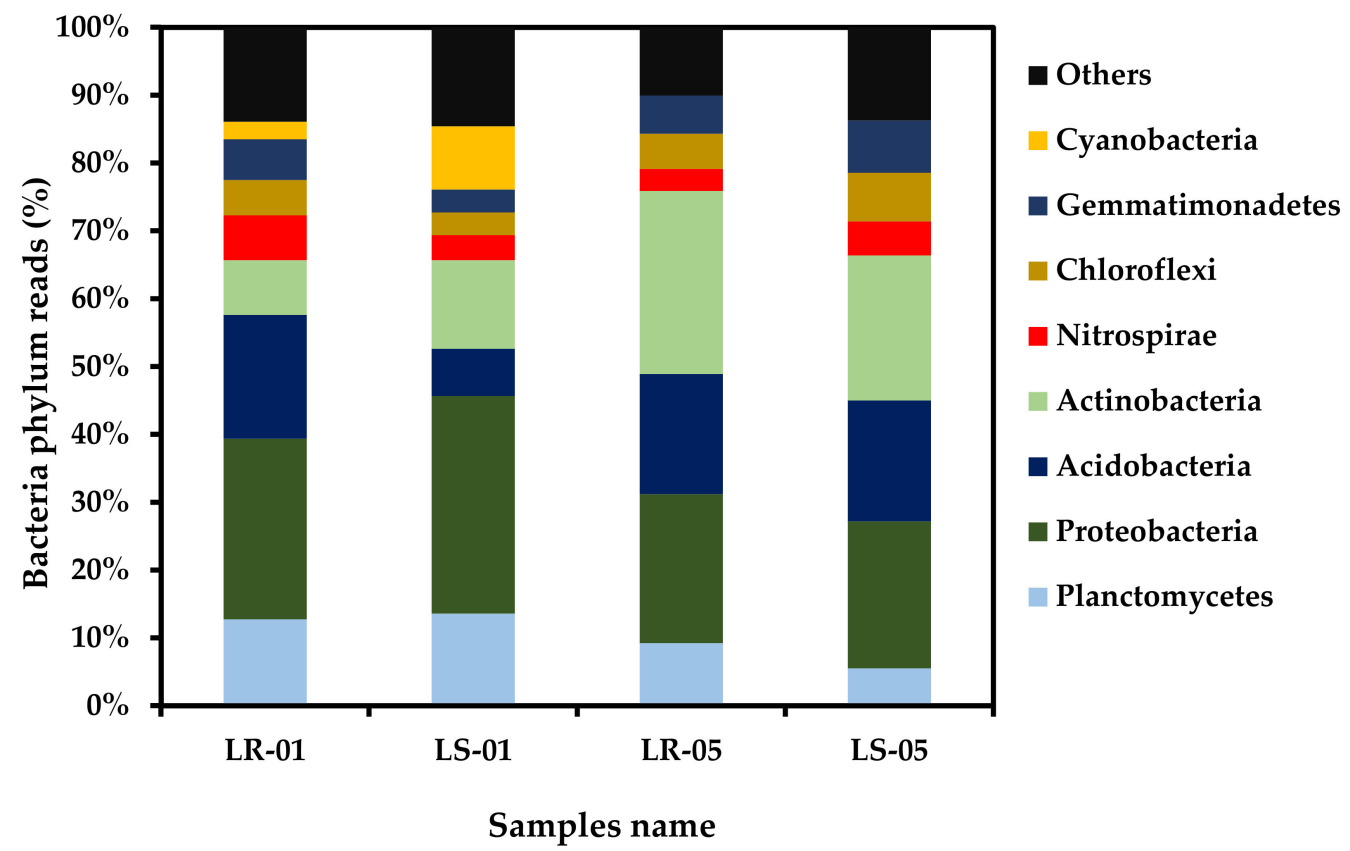

Figure 4. Major bacterial phylum relative abundance of rock and soil samples collected outside the cave (LR-01 and LS-01) and inside the cave (LR-05 and LS-05).

\subsection{Correlation between Predicted Functional Pathways and Carbon Utilization Pattern}

PICRUSt2 was used to predict the potential metabolic functions of the microbial communities in the samples using 16S rRNA amplicon sequence data. The aim of this analysis was to identify the KEGG pathways associated with the metabolism of LMW organic compounds indirectly involved in calcium carbonate formation. The functional prediction analysis revealed the presence of metabolic pathways, including carbohydrates, amino acids, other amino acids, and lipids, or xenobiotic compounds, including energy utilization and membrane transport, both within the samples collected from inside and outside the cave. Among these metabolic pathways, carbohydrate metabolism was found to be the predominant function, followed by amino acid metabolism, in all of the samples, as shown in Figure 5A. However, other metabolic functions, including membrane transport, energy metabolism, xenobiotics biodegradation, metabolism, and lipid metabolism were found in low proportions. Further categorization of the carbohydrate metabolism at KEGG level 
3 indicated the presence of major metabolic functions related to starch, sucrose, galactose, amino sugars, nucleotide sugars, ascorbate, aldarate, the citrate cycle (TCA), and the pentose phosphate pathway in samples collected both inside and outside the cave as shown in Supplementary Table S3. Similarly, amino acid and other amino acid metabolisms were further categorized up to KEGG level 3, which included arginine, proline, phenylalanine, glycine, serine threonine, and glutathione as major functions. Lipid catabolism may be carried out by glycerophospholipid metabolism, while xenobiotic-related metabolism was utilized through bisphenol and benzoate degradation pathways. In addition, the carbon fixation pathway and methane metabolism were the main energy utilization pathways in the diversity structure of the microbiota from inside and outside the cave. Moreover, the $\mathrm{ABC}$ transporter was predominately found as a major function in the membrane transport pathway, which had a relatively higher abundance in the samples collected outside the cave compared to those collected inside the cave (Figure 5B). Furthermore, Pearson's correlation statistical approach was used to evaluate the relationship between extracellular carbon source uptake and the utilization capabilities of the microbial community with their predicted metabolic functions, shown in Figure 6. The correlation analysis revealed that, among all predicted metabolic pathways, starch, sucrose, amino sugars, nucleotide sugars, glycerophospholipid metabolism, and benzoate degradation had a strong positive correlation with extracellular carbon utilization $(p \leq 0.05)$. Additionally, the carbon fixation pathway, methane metabolism, and $\mathrm{ABC}$ transporter were also positively correlated with extracellular carbon metabolic potential. However, several metabolic functions, including photosynthesis antenna proteins, phenylalanine, arginine, proline ascorbate, the aldarate pentose phosphate pathway, and galactose phosphate, were found to be negatively correlated with Biolog EcoPlate ${ }^{\mathrm{TM}}$ carbon substrate utilization.

(A)

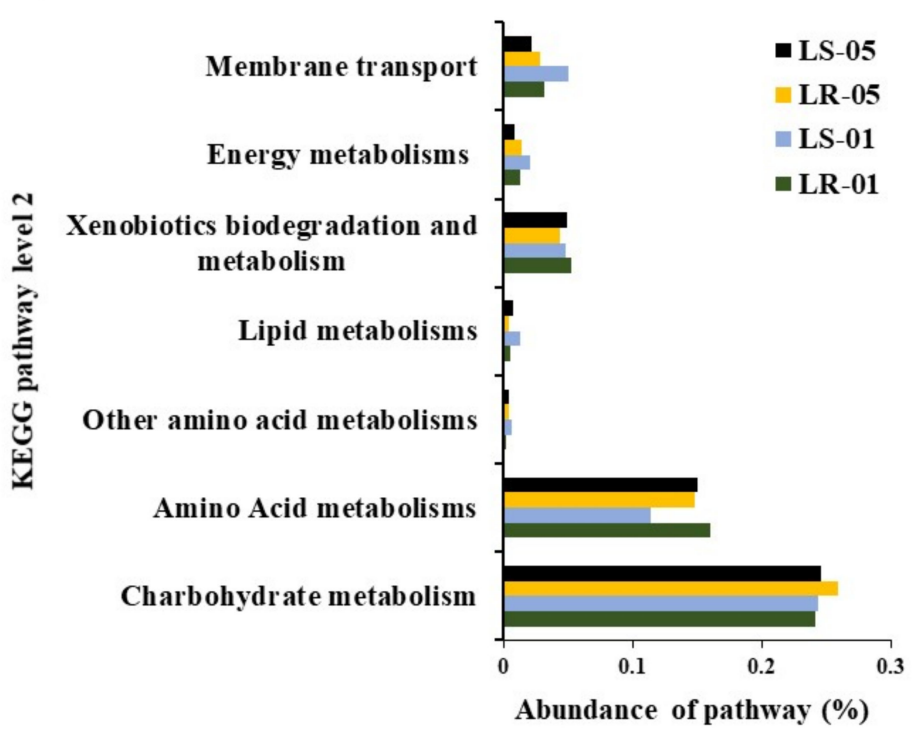

(B)

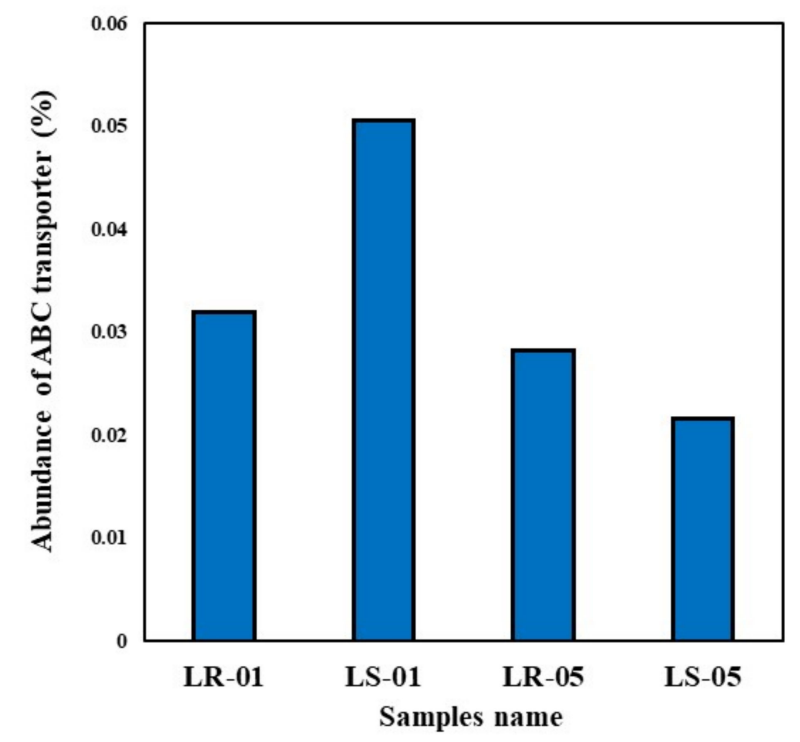

Figure 5. (A) Visualization of predicted major metabolic pathway categories relative to the abundance of each sample, including environmental information processing. (B) Presence of environmental information processing (ABC transporter) genes in samples collected outside and inside the cave using PICRUSt2 analysis. 


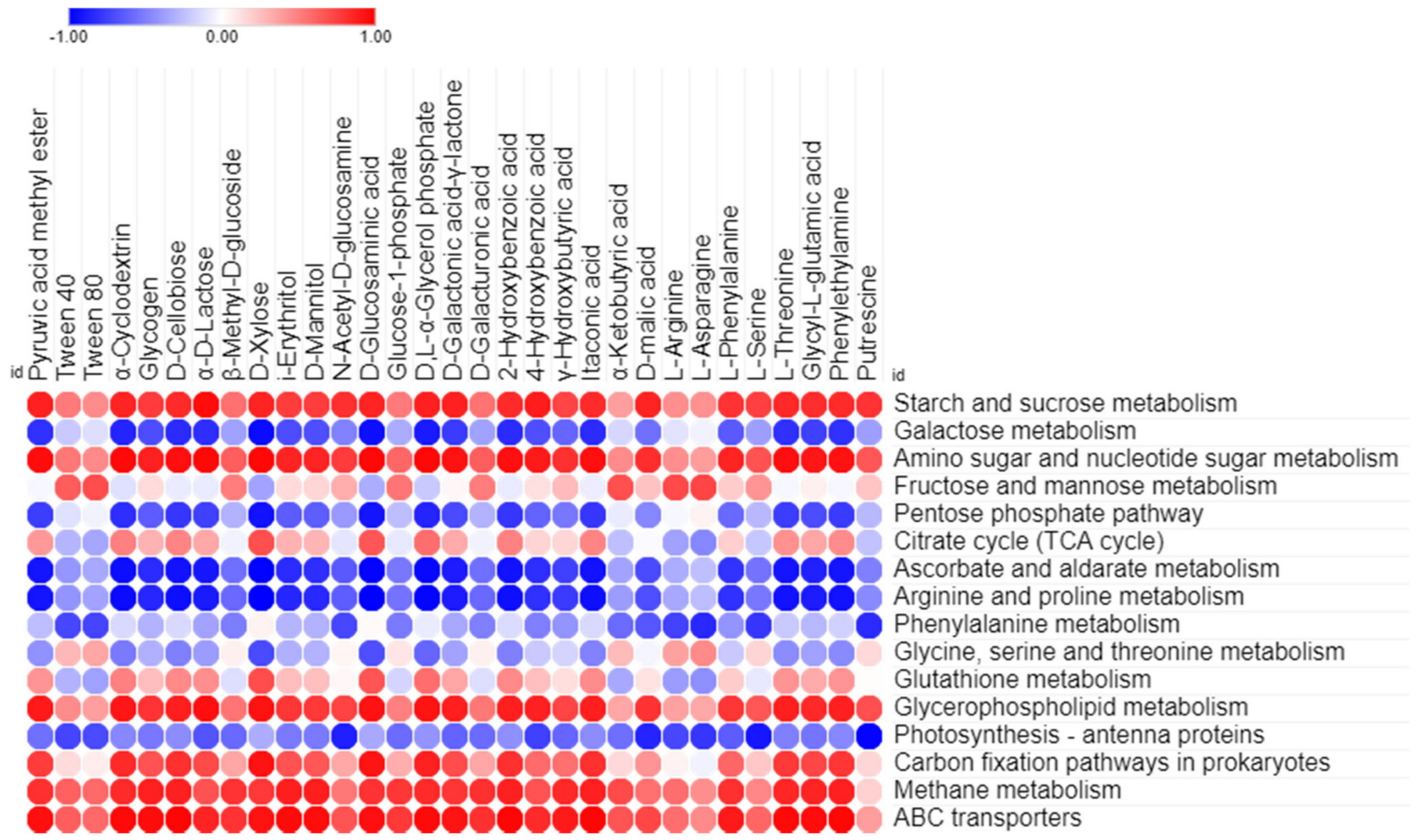

Figure 6. Visualization of Pearson's correlation statistical test between 31 Biolog EcoPlate ${ }^{\mathrm{TM}}$ carbon sources utilization and related predicted functional KEGG pathway abundance of bacterial communities in all over the samples.

\subsection{Visualization of Multivariate Analysis of Eco Plate and $16 \mathrm{~S}$ rRNA Amplicon Data}

PCA analysis was conducted for the bacterial communities found both outside and inside the cave, performed with their major carbon source utilization pattern in Biolog EcoPlate $^{\mathrm{TM}}$ against the incubation period $(168 \mathrm{~h})$ and predicted KEGG metabolic functions associated with carbon metabolism. Planctomycetes, Proteobacteria, Cyanobacteria, and Nitrospirae were strongly associated with the samples collected outside the cave, in which sunlight was a key determining factor (Figure 7). Additionally, the bacteria of this sampling zone were found to be involved in the utilization of the majority of carbon sources. These bacteria were predominantly associated with two important metabolic functions, namely the amino acid and carbohydrate metabolism. However, Acidobacteria, Actinobacteria, Chloroflexi, and Gemmatimonadetes were more closely associated with the soil and rock samples collected inside the cave. In Figure 7, the arrows, which represent the different types of carbon source utilization patterns, were not correlated with sunlight, but rather other environmental parameters, including humidity and temperature. The lipid metabolism, other amino acids, and energy metabolism pathways (e.g., carbon fixation pathways in prokaryotes and methane metabolisms) were found to be closely related with the evolution of the microbial communities and environmental information processing inside the cave. 


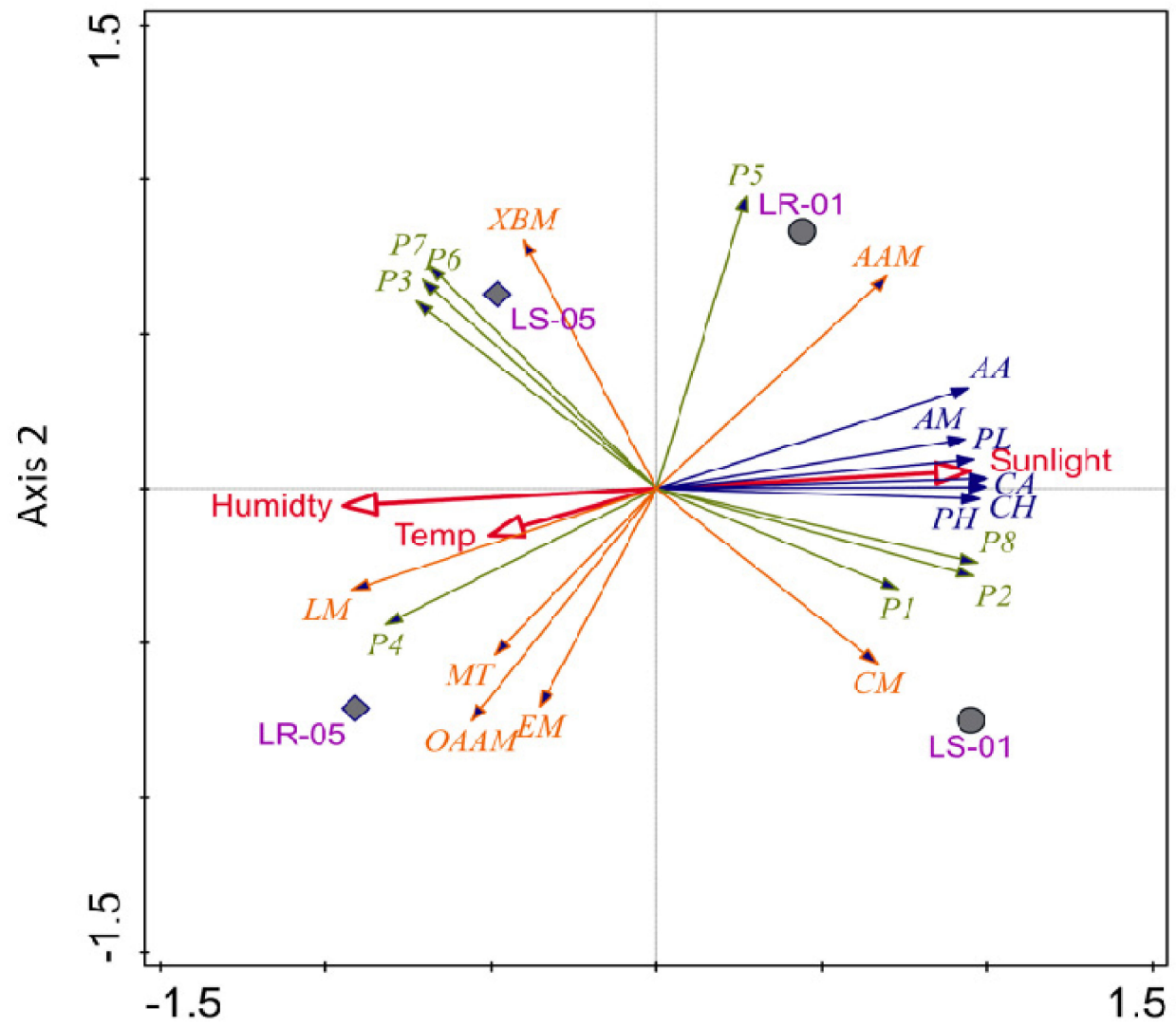

Axis 1

Figure 7. Principle component analysis of major carbon sources (AA, amino acids; AM, amines; PL, polymers; $\mathrm{CA}$, carboxylic acid; $\mathrm{CH}$, carbohydrates; $\mathrm{PH}$, phenols) with predicted KEGG pathways (XBM, Xenobiotic biodegradation and metabolisms; AAM, amino acid metabolism; CM, carbohydrate metabolism; LM, lipid metabolism; EM, energy metabolism; OAAM, other amino acid metabolism; MT, membrane transport), including major bacterial phylum (P1, Planctomycetes; P2, Proteobacteria; P3, Acidobacteria; P4, Actinobacteria; P5, Nitrospirae; P6, Chloroflexi, P7, Gemmatimonadetes; P8, Cyanobacteria) against the environmental parameters.

\section{Discussion}

The use of Biolog EcoPlate ${ }^{\mathrm{TM}}$ is highly convenient to measure community-level microbial catabolic activity and diversity in terms of substrate utilization in a variety of complex environmental samples. However, it has some limitations, such as its ineffectiveness on aquatic samples, the sensitivity of redox dye against temperature, and its efficiency on heterotrophic bacteria [34]. However, in our field of study, it is rarely used and represents a completely novel approach [24]. In the present study, the microbiota from soil and rock samples collected outside of a limestone cave showed the highest rate of AWCD, compared with samples collected inside the cave, which showed a relatively low AWCD curve. In addition, fluctuations in the AWCD curves were also observed in the microbial communities of different sample types (rock and soil) within the same sampling zone. With regards to the difference observed in the AWCD patterns between sampling environments (i.e., outside and inside the cave), the microbial communities found outside of the cave were highly metabolically active, consuming and up taking carbon from the environment to fuel catabolic activity during cell growth. Previous studies have also reported that, in terrestrial environments, sunlight is the most influential factor for the production of simple organic carbon via photodegradation, resulting in a higher metabolic activity in microorganisms $[35,36]$. Similarly, in a study on permafrost, another extreme environment, the authors found that sunlight exposure can enhance the microbial respiration rate up to 
$>40 \%$ compared with dark areas, mediated by dissolved organic carbon [37]. In this context, the absence of solar radiation may be responsible for the low levels of photodegradation within cave environments. This leads to the formation of oligotrophic conditions, which ultimately inhibit organic carbon-mediated microbial functions and the catabolic rate of the microbial communities $[37,38]$. Thus, according to our results, the microbial communities inside caves poorly uptake carbon from external sources, resulting in lower AWCD curves. Additionally, due to their inefficient catabolic activity, these microbial communities may not be able to produce sufficient NADH during respiration, resulting in a reduced tetrazolium dye reduction in the Biolog EcoPlate ${ }^{\mathrm{TM}}$ plate during the 7-day incubation period, and a lower AWCD pattern [39].

In addition to the main six groups of carbon sources utilized by the microbial communities, our results showed that, among 31 carbon substrates, the utilization of carbon from carbohydrates, amino acids, and carboxylic acid groups was markedly higher in the microbial communities outside the cave. Although the microbial communities inside the cave also utilized these carbon substrates, the level of utilization was much lower than that of bacteria outside the cave. These results are in accordance with a previous study on soil microbial ecology, which found that carbohydrates, amino acids, and carboxylic acids belong to the LMW organic substance (LMWOS) class, which are preferentially taken up by bacteria found in soil for cellular activity, producing $\mathrm{CO}_{2}$ as a by-product [40]. These three groups of LMWOS are utilized by bacteria because of their high demand for carbon for anabolic products during cell mass development, which are also incorporated into the citric acid cycle, glycolysis, and the pentose phosphate pathway [29]. However, the dark conditions inside caves hamper sunlight-based primary productivity and the decomposition of these three major classes of LMWOS, leading to low concentrations inside caves, despite the input of allochthonous carbon from outside caves [41]. We suggest that the oligotrophic condition could contribute to the LMWOS stress situation in the inside-cave environment. Therefore, the microbial community of inside cave in this study was not adapted to the utilization of carbohydrates, amino acids, or carboxylic acid groups as carbon sources, and showed a low utilization pattern for these three groups of substrates.

According to Illumina-based $16 \mathrm{~S}$ rRNA amplicon data analysis, Proteobacteria, Acidobacteria, Actinobacteria, and Planctomycetes were the predominant groups of phyla, followed by Nitrospirae, Chloroflexi, and Gemmatimonadetes, across all samples, irrespective of the sampling area. However, Cyanobacteria was the only phylum that was present in both rock and soil samples outside of the cave. Among these major actinobacteria, Acidobacteria, Chloroflexi, and Gemmatimonadetes were mainly associated with sampling inside the cave. This is in accordance with the results of a shotgun metagenomic study on the diversity of the microbial community inside a Manao-Pee cave in Thailand. This study found that actinobacteria and proteobacteria were predominantly associated with the limestone sample. However, in their study, Bacteroidetes, Firmicutes, Acidobacteria, Planctomycetes, Chloroflexi, Gemmatimonadetes, and Cyanobacteria were less abundant [15]. Additionally, another previous study based on metagenomic analysis of the microbial community of limestone reported a phylum level distribution and abundance similar to that found in the present study [42]. In addition, Meier et al., (2017) also found that a similar distribution pattern of microbial diversity at the phylum level associated with the microbial communities of soil and rock samples in regions rich in limestone. They found that Proteobacteria was predominant in the soil samples, while Proteobacteria, Actinobacteria, Bacteroidetes, and Firmicutes were predominant in the rock samples [43]. In the present study, among the identified phyla, Actinobacteria, Acidobacteria, Chloroflexi, and Gemmatimonadetes were found to be negatively correlated with sunlight, suggesting that these microbes use light-independent metabolic pathways. Microorganisms associated with dark and nutrient-poor cave areas are able to survive easily in this type of environment due to their chemoautotrophic mechanisms [44]. In fact, the majority of bacterial communities found in extreme environments are associated with nitrogen-, sulfur-, and methane-based chemoautotrophic pathways [45]. 
According to functional prediction analysis, the abundance of LMW organic compounds related metabolic pathways reads such as carbohydrates, amino acids, other amino acids, and lipids were predominating across all samples irrespective of the sampling area. This result suggests that microbial communities are able to utilize organic carbon sources for their metabolism. Previous studies have also demonstrated that the carbohydrate and amino acid metabolisms are the most dominant metabolic pathways associated with the microbial communities found in caves [15,27]. The higher contribution of these LMW organic compounds to the metabolic pathways of soil and rock samples collected outside the cave demonstrates that these were more functional than those from inside the cave, which is reflected by the utilization of the carbon sources from the Biolog EcoPlate ${ }^{\mathrm{TM}}$ by the different bacterial communities. Conversely, even though the bacterial communities inside the cave also had LMW organic compounds-related metabolic pathways, they did not show high levels of utilization activity for the different carbon sources in the Biolog EcoPlate ${ }^{\mathrm{TM}}$ assays. This suggests that oligotrophic situations inside caves ecosystems might be LMW organic compound-related metabolic pathways that are non-functional in real cases. Previous studies have found that the ABC transporter, which plays a key role in the uptake of LMW organic compounds from the environment, was relatively less abundant among the microbial communities found inside caves, which may explain their lower AWCD curves [46,47]. In addition, the methane-linked energy metabolic pathway, including a small amount of carbon fixation pathways, was found to be predominant in the bacterial communities of the cave samples. This could imply that bacteria found in caves can use methane as an alternative source of carbon, engaging in primary production via the chemolithoautotrophic pathway [15]. Previous studies have also shown that ABC transporter-associated genes are expressed at comparatively lower levels in autotrophic bacteria [48,49]. In this context, this study also considered the chemolithoautotrophic pathway, which is an autotropic pathway, dominant in the bacterial communities of inside-cave samples.

\section{Conclusions}

This study demonstrated that environmental factors greatly influence the taxonomic and physiological distribution of microbial communities in extreme ecosystems, such as limestone caves. These communities of bacteria use alternative sources of micronutrients from their environment for cellular growth, and ultimately participate in primary production to support higher-order organisms. The results of the Biolog EcoPlate ${ }^{\mathrm{TM}}$ assays showed that the microbial communities outside caves consumed simpler forms of different carbon sources, such as carbohydrates, amino acids, and carboxylic acids, than those inside caves. In contrast, specific bacterial groups, such as Actinobacteria, Proteobacteria, and Acidobacteria, could adapt to oligotrophic conditions by using alternative energy metabolic pathways, collectively known as chemoautotrophs, and were found to be the dominant phyla inside the caves. In the present study, the methane-based chemoautotrophic pathway was found to play a leading role in primary production by these bacterial communities, while the LMW organic compound-dependent primary production was probably nonfunctional in the dark oligotrophic conditions of inside-cave areas.

Supplementary Materials: The following are available online at https: / www.mdpi.com/article/ 10.3390/microorganisms9081789/s1, Figure S1: Abundance of bacteria phylum in all rock and samples Figure S2: Heatmap analysis with dendrogram plot at the genus level for all samples, Table S1: Details of sampling environmental conditions, Table S2: Pearson's correlation test against predominant bacteria phylum with environmental factors, Table S3: KEGG pathway normalized reads for rock and soil samples collected outside and inside the cave, Table S4: Primer information list, Table S5: Microbial community beta diversity index. 
Author Contributions: Conceptualization, S.K., B.-M.H., C.-W.T. and J.-S.C.; methodology, C.-W.F., B.H. and T.-H.C.; software, V.N., S.K. and J.-S.C.; validation, C.-W.F., C.-W.T., J.-S.C. and B.-M.H.; formal analysis, S.K. and T.-H.C.; investigation, S.K., B.H. and C.-W.F.; resources, J.-S.C. and B.-M.H.; data curation, V.N., S.K. and C.-W.T.; writing-original draft preparation, S.K., C.-W.T. and J.-S.C.; writing-review and editing, B.-M.H.; visualization, B.H., V.N. and C.-W.F.; supervision, S.K., C.-W.T. and J.-S.C.; project administration, B.-M.H.; funding acquisition, C.-W.T. and B.-M.H. All authors have read and agreed to the published version of the manuscript.

Funding: This research was funded by the Ministry of Science and Technology of Taiwan (MOST 109-2116-M-194-013-) and Ditmanson Medical Foundation Chia-Yi Christian Hospital-Nation Chung Cheng University Joint Research Program (CYCH-CCU-2021-05; R110-26).

Institutional Review Board Statement: Not applicable.

Informed Consent Statement: Not applicable.

Data Availability Statement: The data presented in this study are available on request from the corresponding author.

Acknowledgments: This research was thanks for the Center for Innovative Research on Aging Society (CIRAS) from The Featured Areas Research Center Program within the framework of the Higher Education Sprout Project by the Ministry of Education (MOE) in Taiwan.

Conflicts of Interest: The authors declare no conflict of interest.

\section{References}

1. Seifan, M.; Berenjian, A. Microbially induced calcium carbonate precipitation: A widespread phenomenon in the biological world. Appl. Microbiol. Biotechnol. 2019, 103, 4693-4708. [CrossRef]

2. Reddy, M.S. Biomineralization of calcium carbonates and their engineered applications: A review. Front. Microbiol. $2013,4,314$.

3. Kim, Y.; Roh, Y. Microbially Induced Carbonate Precipitation Using Microorganisms Enriched from Calcareous Materials in Marine Environments and Their Metabolites. Minerals 2019, 9, 722. [CrossRef]

4. Anbu, P.; Kang, C.-H.; Shin, Y.-J.; So, J.-S. Formations of calcium carbonate minerals by bacteria and its multiple applications. SpringerPlus 2016, 5, 1-26. [CrossRef]

5. Haiming, T.; Xiaoping, X.; Chao, L.; Xiaochen, P.; Kaikai, C.; Weiyan, L.; Ke, W. Microbial carbon source utilization in rice rhizosphere and nonrhizosphere soils with short-term manure $\mathrm{N}$ input rate in paddy field. Sci. Rep. 2020, 10, 6487-6489. [CrossRef]

6. Waschina, S.; D'Souza, G.G.; Kost, C.; Kaleta, C. Metabolic network architecture and carbon source determine metabolite production costs. FEBS J. 2016, 283, 2149-2163. [CrossRef]

7. Chen, M.; Qiu, T.; Sun, Y.; Song, Y.; Wang, X.; Gao, M. Diversity of tetracycline- and erythromycin-resistant bacteria in aerosols and manures from four types of animal farms in China. Environ. Sci. Pollut. Res. 2019, 26, 24213-24222. [CrossRef]

8. Sutfin, N.A.; Wohl, E.E.; Dwire, K.A. Banking carbon: A review of organic carbon storage and physical factors influencing retention in floodplains and riparian ecosystems. Earth Surf. Process. Landf. 2015, 41, 38-60. [CrossRef]

9. Babur, E.; Dindaroglu, T. Seasonal Changes of Soil Organic Carbon and Microbial Biomass Carbon in Different Forest Ecosystems. In Environmental Factors Affecting Human Health; IntechOpen: London, UK, 2020.

10. DeJong, J.T.; Soga, K.; Banwart, S.A.; Whalley, W.R.; Ginn, T.R.; Nelson, D.C.; Mortensen, B.M.; Martinez, B.C.; Barkouki, T. Soil engineering in vivo: Harnessing natural biogeochemical systems for sustainable, multi-functional engineering solutions. J. R. Soc. Interface 2011, 8, 1-15. [CrossRef] [PubMed]

11. Chi, J.; Zhang, W.; Wang, L.; Putnis, C.V. Direct Observations of the Occlusion of Soil Organic Matter within Calcite. Environ. Sci. Technol. 2019, 53, 8097-8104. [CrossRef]

12. Ondrasek, G.; Begić, H.B.; Zovko, M.; Filipović, L.; Meriño-Gergichevich, C.; Savic, R.; Rengel, Z. Biogeochemistry of soil organic matter in agroecosystems \& environmental implications. Sci. Total Environ. 2018, 658, 1559-1573. [CrossRef]

13. Van Hees, P.A.; Jones, D.L.; Finlay, R.; Godbold, D.L.; Lundström, U.S. The carbon we do not see-The impact of low molecular weight compounds on carbon dynamics and respiration in forest soils: A review. Soil Biol. Biochem. 2005, 37, 1-13. [CrossRef]

14. Cotrufo, M.F.; Soong, J.L.; Horton, A.J.; Campbell, E.E.; Haddix, M.; Wall, D.H.; Parton, W.J. Formation of soil organic matter via biochemical and physical pathways of litter mass loss. Nat. Geosci. 2015, 8, 776-779. [CrossRef]

15. Wiseschart, A.; Mhuantong, W.; Tangphatsornruang, S.; Chantasingh, D.; Pootanakit, K. Shotgun metagenomic sequencing from Manao-Pee cave, Thailand, reveals insight into the microbial community structure and its metabolic potential. BMC Microbiol. 2019, 19, 1-14. [CrossRef] [PubMed]

16. Chen, Y.; Wu, L.; Boden, R.; Hillebrand, A.; Kumaresan, D.; Moussard, H.; Baciu, M.; Lu, Y.; Murrell, J.C. Life without light: Microbial diversity and evidence of sulfur- and ammonium-based chemolithotrophy in Movile Cave. ISME J. 2009, 3, $1093-1104$. [CrossRef] 
17. Wang, J.; Ersan, Y.C.; Boon, N.; De Belie, N. Application of microorganisms in concrete: A promising sustainable strategy to improve concrete durability. Appl. Microbiol. Biotechnol. 2016, 100, 2993-3007. [CrossRef] [PubMed]

18. Zhu, T.; Dittrich, M. Carbonate precipitation through microbial activities in natural environment, and their potential in biotechnology: A review. Front. Bioeng. Biotechnol. 2016, 4, 4. [CrossRef] [PubMed]

19. Castanier, S.; Le Métayer-Levrel, G.; Perthuisot, J.-P. Bacterial Roles in the Precipitation of Carbonate Minerals. In Microbial Sediments; Springer: Berlin/Heidelberg, Germany, 2000; pp. 32-39. [CrossRef]

20. Ortiz, M.; Legatzki, A.; Neilson, J.W.; Fryslie, B.; Nelson, W.M.; Wing, R.; Soderlund, C.A.; Pryor, B.M.; Maier, R. Making a living while starving in the dark: Metagenomic insights into the energy dynamics of a carbonate cave. ISME J. 2013, 8, 478-491. [CrossRef]

21. Feigl, V.; Ujaczki, E.; Vaszita, E.; Molnár, M. Influence of red mud on soil microbial communities: Application and comprehensive evaluation of the Biolog EcoPlate approach as a tool in soil microbiological studies. Sci. Total Environ. 2017, 595, 903-911. [CrossRef]

22. Salomo, S.; Münch, C.; Röske, I. Evaluation of the metabolic diversity of microbial communities in four different filter layers of a constructed wetland with vertical flow by Biolog ${ }^{\mathrm{TM}}$ analysis. Water Res. 2009, 43, 4569-4578. [CrossRef]

23. Grządziel, J.; Furtak, K.; Gałąka, A. Community-Level Physiological Profiles of Microorganisms from Different Types of Soil That are Characteristic to Poland-A Long-Term Microplot Experiment. Sustainability 2018, 11, 56. [CrossRef]

24. Gryta, A.; Frąc, M.; Oszust, K. The Application of the Biolog EcoPlate Approach in Ecotoxicological Evaluation of Dairy Sewage Sludge. Appl. Biochem. Biotechnol. 2014, 174, 1434-1443. [CrossRef]

25. Chan, C.S.; Chan, K.-G.; Tay, Y.-L.; Chua, Y.-H.; Goh, K.M. Diversity of thermophiles in a Malaysian hot spring determined using 16S rRNA and shotgun metagenome sequencing. Front. Microbiol. 2015, 6, 177. [CrossRef]

26. D'Auria, G.; Artacho, A.; Rojas, R.A.; Bautista, J.S.; Méndez, R.; Gamboa, M.T.; Gamboa, J.R.; Gómez-Cruz, R. Metagenomics of Bacterial Diversity in Villa Luz Caves with Sulfur Water Springs. Genes 2018, 9, 55. [CrossRef]

27. De Mandal, S.; Chatterjee, R.; Kumar, N.S. Dominant bacterial phyla in caves and their predicted functional roles in C and N cycle. BMC Microbiol. 2017, 17, 90. [CrossRef] [PubMed]

28. Young, E.; Carey, M.; Meharg, A.A.; Meharg, C. Microbiome and ecotypic adaption of Holcus lanatus (L.) to extremes of its soil $\mathrm{pH}$ range, investigated through transcriptome sequencing. Microbiome 2018, 6, 48. [CrossRef] [PubMed]

29. Kenarova, A.; Radeva, G.; Traykov, I.; Boteva, S. Community level physiological profiles of bacterial communities inhabiting uranium mining impacted sites. Ecotoxicol. Environ. Saf. 2014, 100, 226-232. [CrossRef] [PubMed]

30. Grządziel, J.; Gałązka, A. Microplot long-term experiment reveals strong soil type influence on bacteria composition and its functional diversity. Appl. Soil Ecol. 2018, 124, 117-123. [CrossRef]

31. Bokulich, N.A.; Subramanian, S.; Faith, J.J.; Gevers, D.; Gordon, J.I.; Knight, R.; Mills, D.A.; Caporaso, J.G. Quality-filtering vastly improves diversity estimates from Illumina amplicon sequencing. Nat. Methods 2013, 10, 57-59. [CrossRef]

32. Bolyen, E.; Rideout, J.R.; Dillon, M.R.; Bokulich, N.A.; Abnet, C.C.; Al-Ghalith, G.A.; Alexander, H.; Alm, E.J.; Arumugam, M.; Asnicar, F.; et al. Reproducible, interactive, scalable and extensible microbiome data science using QIIME 2. Nat. Biotechnol. 2019, 37, 852-857. [CrossRef]

33. DouglasGM, M.; ZaneveldJ, Y.; BrownJR, T.; HuttenhowerC, L. PICRUSt2: An improved and customizable approach for metagenome inference. bioRxiv 2020. [CrossRef]

34. Kurten, G.L.; Barkoh, A. Evaluation of Community-Level Physiological Profiling for Monitoring Microbial Community Function in Aquaculture Ponds. N. Am. J. Aquac. 2015, 78, 34-44. [CrossRef]

35. Bais, A.F.; Lucas, R.M.; Bornman, J.F.; Williamson, C.E.; Sulzberger, B.; Austin, A.; Wilson, S.R.; Andrady, A.L.; Bernhard, G.; McKenzie, R.L.; et al. Environmental effects of ozone depletion, UV radiation and interactions with climate change: UNEP Environmental Effects Assessment Panel, update 2017. Photochem. Photobiol. Sci. 2018, 17, 127-179. [CrossRef]

36. Pieristè, M.; Forey, E.; Sahraoui, A.L.-H.; Meglouli, H.; Laruelle, F.; Delporte, P.; Robson, T.M.; Chauvat, M. Spectral Composition of Sunlight Affects the Microbial Functional Structure of Beech Leaf Litter During the Initial Phase of Decomposition. Plant Soil 2020, 451, 515-530. [CrossRef]

37. Ward, C.P.; Nalven, S.G.; Crump, B.C.; Kling, G.W.; Cory, R.M. Photochemical alteration of organic carbon draining permafrost soils shifts microbial metabolic pathways and stimulates respiration. Nat. Commun. 2017, 8, 1-8. [CrossRef]

38. Zhu, H.-Z.; Zhang, Z.-F.; Zhou, N.; Jiang, C.-Y.; Wang, B.-J.; Cai, L.; Liu, S.-J. Diversity, Distribution and Co-occurrence Patterns of Bacterial Communities in a Karst Cave System. Front. Microbiol. 2019, 10, 1726. [CrossRef] [PubMed]

39. Bochner, B.R. Global phenotypic characterization of bacteria. FEMS Microbiol. Rev. 2008, 33, 191-205. [CrossRef]

40. Gunina, A.; Smith, A.R.; Kuzyakov, Y.; Jones, D. Microbial uptake and utilization of low molecular weight organic substrates in soil depend on carbon oxidation state. Biogeochemistry 2017, 133, 89-100. [CrossRef]

41. Ravn, N.R.; Michelsen, A.; Reboleira, A.S. Decomposition of Organic Matter in Caves. Front. Ecol. Evol. 2020, 8. [CrossRef]

42. Wu, Y.; Tan, L.; Liu, W.; Wang, B.; Wang, J.; Cai, Y.; Lin, X. Profiling bacterial diversity in a limestone cave of the western Loess Plateau of China. Front. Microbiol. 2015, 6, 244. [CrossRef] [PubMed]

43. Meier, A.; Singh, M.; Kastner, A.; Merten, D.; Büchel, G.; Kothe, E. Microbial communities in carbonate rocks-from soil via groundwater to rocks. J. Basic Microbiol. 2017, 57, 752-761. [CrossRef] [PubMed]

44. Tomczyk-Żak, K.; Zielenkiewicz, U. Microbial Diversity in Caves. Geomicrobiol. J. 2015, 33, 20-38. [CrossRef] 
45. Kumaresan, D.; Wischer, D.; Stephenson, J.; Hillebrand-Voiculescu, A.; Murrell, J.C. Microbiology of Movile Cave-A Chemolithoautotrophic Ecosystem. Geomicrobiol. J. 2014, 31, 186-193. [CrossRef]

46. Cui, J.; Davidson, A.L. ABC solute importers in bacteria. Essays Biochem. 2011, 50, 85-99. [CrossRef]

47. Zheng, Q.; Lu, J.; Wang, Y.; Jiao, N. Genomic reconstructions and potential metabolic strategies of generalist and specialist heterotrophic bacteria associated with an estuary Synechococcus culture. FEMS Microbiol. Ecol. 2019, 95, fiz017. [CrossRef]

48. Bergauer, K.; Fernandez-Guerra, A.; Garcia, J.A.L.; Sprenger, R.; Stepanauskas, R.; Pachiadaki, M.; Jensen, O.N.; Herndl, G.J. Organic matter processing by microbial communities throughout the Atlantic water column as revealed by metaproteomics. Proc. Natl. Acad. Sci. USA 2017, 115, E400-E408. [CrossRef]

49. Ren, Q.; Paulsen, I.T. Large-Scale Comparative Genomic Analyses of Cytoplasmic Membrane Transport Systems in Prokaryotes. J. Mol. Microbiol. Biotechnol. 2007, 12, 165-179. [CrossRef] 